\title{
Blockchain is dead! Long live Blockchain!
}

\section{Joshua Ellul}

Centre for Distributed Ledger Technologies, University of Malta

Correspondence: joshua.ellul@um.edu.mt

Received: 16 January 2021 Accepted: 14 March 2021 Published: 25 March 2021

\begin{abstract}
A decade on since Satoshi's Bitcoin paper, Blockchain is now considered to be sliding into the trough of Gartner's hype cycle. Claims in regard to Blockchain and Cryptocurrencies being dead are on the rise, whilst at the same time many claim the contrary. The vague statement encapsulates many different aspects and perspectives of a myriad of use cases, technology and platforms including both the technique as a whole and as individual instantiations.

In this paper, we unpack the statement, break it down and investigate objectively concrete factors which provide indication in regard to whether Blockchain is dead. We examine metrics including budgets and investment; company registries and data; community engagement, projects and source code repositories; academic research and programmes; social media posts; and public interest. We individually demonstrate metrics that indicate the respective measures' healthy activity and come to the conclusion that the collective statement 'Blockchain is dead' does not hold. A clear message extracted from the work proposed herein is that success is achieved where the community comes together rather than works in isolation.
\end{abstract}

Keywords: blockechain, ecosystem, research, investment, analysis

JEL Classifications: $A 12$

\section{Introduction}

'Blockchain is dead?'i - a question or statement which many have asked or claimed since the (first) 2017 rise and fall of Bitcoin and other cryptocurrencies. Is it the case that the blockchain field and related sectors are indeed dead? According to Gartner blockchain is now sliding into its hype cycle's Trough of Disillusionmenti in 2020 - depicted and expanded on in Figure 1. Will the technology make its way up the Slope of Enlightenment or will it exit towards its death? To answer the question of whether or not blockchain is dead and/or on its way there, we first need to understand what we mean by it and how we can determine the answer. In this paper, we aim to provide insight in regard to whether this is the case by investigating a number of different facets of the blockchain sector.

Whilst, death implies a permanent state of inactivity, a looser meaning will be used to determine whether or not blockchain is dead - if activity within the sector is drastically reduced (even if temporary) then for the sake of reaching a conclusion in the current period under investigation it will be assumed that it is dead or on its deathbed.

To determine this, activities within various facets of the blockchain sector will be investigated including cryptocurrencies, smart contracts, distributed ledger technology (DLT) and popular platforms including Bitcoin, Ethereum and Hyperledger. Activity of the following vitals will be looked into: (i) new companies being founded; (ii) investment into start-ups and companies; (iii) patents published; (iv) academic papers published; (v) research and development funding; (vi) online search trends; (vii) mining infrastructure and hash rates; and (viii) blockchain-related software development effort.

Indeed, the blockchain sector includes many applications beyond cryptocurrencies [1], and by including cryptocurrencies (and associated hype surrounding them) in this study, the results are influenced beyond what is otherwise pertaining only to the non-cryptocurrency blockchain sector. However, in this first study, the aim is to look at the sector at the most abstract level (including cryptocurrencies).

It would be ideal to go into greater detail for each facet investigated; however, due to space limitation, initial insights in regard to the various aspects will be provided and deeper analysis of each aspect will be left for future work.

A lot of hype surrounded the sector when cryptocurrency prices had surged mid- to late 2017. This hype was short-lived and prices soon came crashing down. Many associate the surge and crash in price with the sector's position in Gartner's hype 


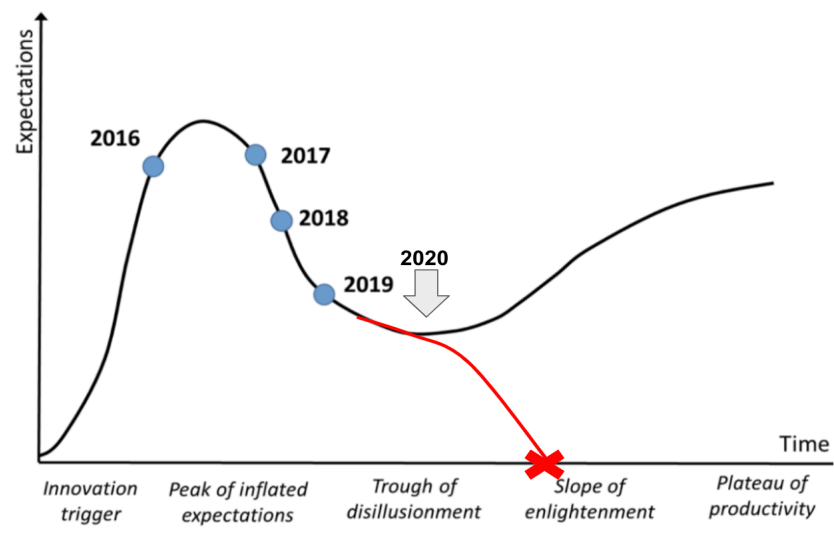

Figure 1: The Gartner Hype Cycle reproduced from [2] and modified.

cycle - yet at the time of writing this paper a second surge in price of cryptocurrencies is being seen. In this paper the period from January 2017 to December 2020 will be used in undertaking the various data gathering and analysis required. Figure 2 depicts the prices of two popular cryptocurrencies, Bitcoin and Ether, for the aforementioned period. The hype period referred to can be seen starting around mid- to late 2017. Whilst, reference will be made to this hype period throughout the paper, in no way is the paper claiming that the Blockchain sector is dependent on cryptocurrencies' success solely (it is but just one factor) and indeed the Blockchain sector may survive independent of cryptocurrencies' success however, reference is made to this period so as to be able to provide initial insight on potential correlations with the period.

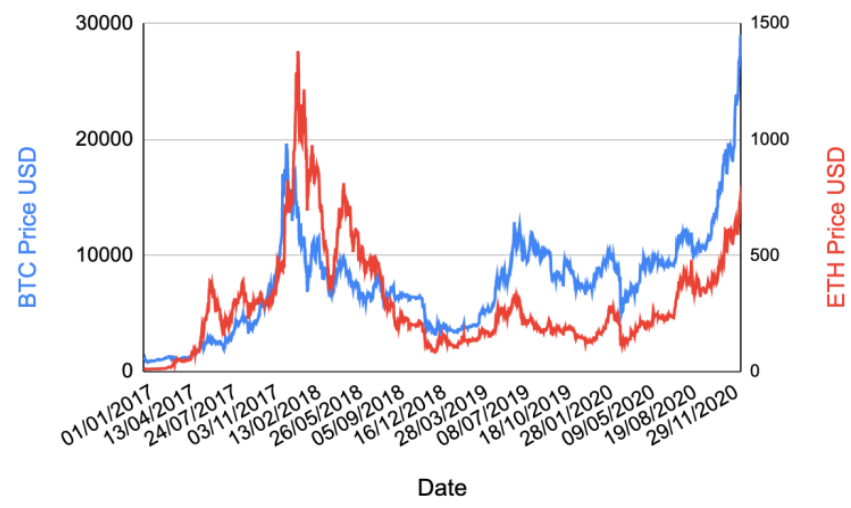

Figure 2: Bitcoin (BTC) and Ether (ETC) Price in United States Dollars (USD).

The paper will now follow by providing insight into the 8 aspects of blockchain activity mentioned above, where each section will describe its purpose, methodology-related aspects and results.

\section{Companies Founded}

Companies founded and services launched are indicators that demonstrate the private sector's belief in a technology's potential. Whilst it has proven difficult to find a registry or list of services which specify launch dates and activity, information relating to companies were retrieved from Crunchbase, iii a publicly available and browsable database providing information about start-ups, companies and their financing [3]. It has been described as 'the premier data asset on the tech/startup world'. iv An exercise was undertaken to determine the number of companies founded between 2017 and 2020 to provide an indication whether an increase or decrease in activity within the sector can be identified.

\section{Methodology}

Some companies in the dataset are not attributed with the full date they were founded but only the year - which end up being associated with $1^{\text {st }}$ January of the respective year. It was decided to remove the companies listed as being founded on $1^{\text {st }}$ January of each year since it would be impossible to identify which of those companies were actually founded on $1^{\text {st }}$ January and which were founded during some other time in the respective year. Also, given that $1^{\text {st }}$ January is a public or bank holiday in many countries it is unlikely that a high number of companies were founded on $1^{\text {st }}$ January.

The data was gathered on 5 January 2021. All company data was retrieved for companies whose descriptions includes any of the following keywords and terms: 'blockchain', 'cryptocurrency', 'cryptocurrencies', 'DLT', 'DLTs', 'distributed ledger technology', 'distributed ledger technologies', 'bitcoin', 'ethereum', 'hyperledger', 'smart contract', 'smart contracts', 'cryptocurrency exchange' and 'crypto exchange'.

\section{Results}

Figure 3 depicts the number of blockchain-related companies (as per the terms listed above) founded per month between January 2017 and December 2020. The numbers show a peak of companies founded between late 2017 and early 2018 which coincides with the hype seen during that period. The question however is whether the post-hype data reflects an indication in regard to whether interest has been completely lost or not in the space. On initial glance one may conclude that the diminishing number of companies founded per month may indicate this.
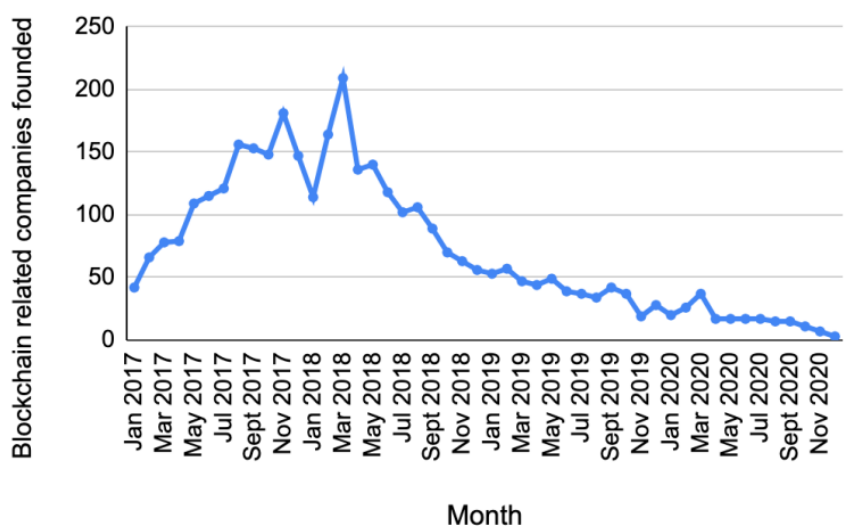

Figure 3: Blockechain-related companies founded between January 2017 and December 2020. 
However, given how Crunchbase collects and processes data especially due to its crowdsourced nature of data collection, 'there is therefore a certain delay between the foundation of the company and its actual registration on Crunchbase' [4]. A company is likely to be listed on Crunchbase 'when it starts looking for investment, or has become part of the portfolio of an investor, or more generally wishes to gain greater visibility online' [4]. However, these numbers should be revisited in a year's time to see if 2020 s numbers are around the numbers currently reported for 2019 to give an indication in regard to increasing or decreasing numbers of companies being founded.

To further support this argument, besides the depth to which this was discussed in [4], the same exercise was conducted for companies categorised under the keyword 'software' - a term that is likely to not have suffered from hype over the past few years. Figure 4 below provides support for this argument that the decreasing number of registered companies does not necessarily mean that companies are not being founded, just that they are not yet listed in the platform. However, what we can conclude is that figures currently reported for blockchainrelated companies founded in 2019 were around the levels of companies founded prior to 2017's hype - and these figures should be revisited in a year's time.

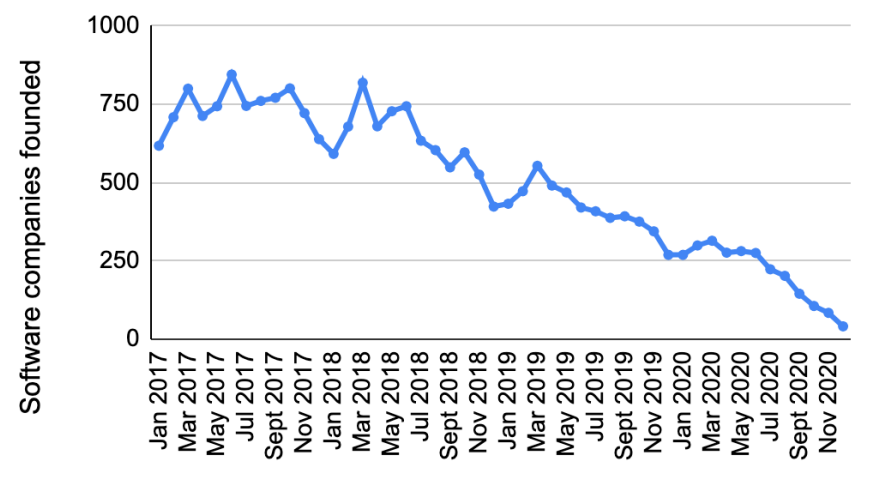

Month

Figure 4: Companies categorised under 'software' founded between January 2017 and December 2020.

Whilst, it would be useful to see how many of these companies are still operating, and whilst Crunchbase data does indicate whether a company has closed, the figures are low and likely not representative of companies actually closed (likely due to the crowdsourced nature of data). The figures reported for closed companies for years 2017, 2018, 2019 and 2020 are 64, 40, 6 and 1, respectively. Whilst, accurate figures regarding whether companies are still operating would help to determine a more objective view of the success of the industry, the aim of this exercise highlights continual renewed interest to start companies in the sector, which is an indicator (albeit weaker) of the sectors potential success. That said, the general consensus is that many companies/projects initiated during the hype 'failed to materialize' [5], as would be the case for any initially hyped technology (just like the dotcom bubble). Whilst some originally cite a main problem being that blockchain is 'innovative technology in search for use cases' [6] Navqi and Hussain [5] highlight main problems which focus on the lack of applying an evidence-based practice approach. In their study 517 projects and start-up companies were analysed and their results clearly indicate that minimal evidence was used to establish whether a project's problem was actually a problem that needed solving.

Out of the 2778, 2552, 757 and 301 companies founded retrieved from Crunchbase for 2017, 2018, 2019 and 2020, respectively, 265, 259, 103 and 33 were companies that were listed as being from the following industries: private cloud, cloud infrastructure, cloud computing, cloud management, cloud storage, cloud data services, cloud security, artificial intelligence, machine learning, internet of things and quantum computing. That is around $10 \%$ of companies founded each year. This demonstrates the technology's cross pollination into other sectors which may also be seen as a testament to its future potential within other sectors.

The results heeded indeed provide a single holistic view including all cryptocurrency-related companies and other noncryptocurrency blockchain companies together. The 2017 hype likely resulted in many cryptocurrency-based companies being founded the same and following year, which may drown out figures relating to those companies focused only on blockchain beyond cryptocurrencies. However, nonetheless cryptocurrency-focused companies also play a part within the sector and therefore it was decided to report the results in this manner. In future it could very well be that the number of companies founded are seen to be much less since interest to start a cryptocurrency after the hype ended may have diminished (though a second wave of interest in cryptocurrencies is being seen at the time of writing). Nonetheless, future work should go into further depth in regard to interest in the various sub-sectors (e.g. cryptocurrencies, supply chain applications, enterprise blockchain solutions, etc).

\section{Investment}

Amounts of investment raised are good indicators to identify technologies that have potential since entities (venture capitalists, investors, etc.) specialised in determining what technologies have potential, literally bet their money on the respective technology. Therefore, data has been gathered to determine how funding and investments have fared over the period.

Crunchbase is often claimed to be 'a primary data source for investors' [7] which may be due to its large investor network comprising of at least 3,000 global investment firms who 'submit monthly portfolio updates' [7]. Therefore, investments over the period will be analysed to provide insight with respect to this study. Since Crunchbase crowdsources its data, it was decided to also compare investment data from CB Insights ${ }^{v}$ [8]. 


\section{Methodology}

For data retrieved from Crunchbase, the same keywords and terms used in the previous section were used to filter out the different investments made to companies whose descriptions included the terms. The funding round datasets included exact dates for investment periods (unlike company foundation dates). The data was also gathered on 5 January 2021, and the investments were aggregated according to the month. For data retrieved from CB Insights, investments (categorised under deals) were retrieved from companies categorised under 'Blockchain' (i.e. in the 'Blockchain' collection) for the following investment stages: Seed / Angel, Series A, Series B, Series C, Series D, Series E+, Private Equity, Growth Equity, Other Venture Capital, and IPO. Data was retrieved from CB Insights on 6 March 2021.

\section{Results}

For data retrieved from Crunchbase, to get an overview of funding in the sector, all types of funding rounds were included - from pre-seed and seed funding, to all the different series funding and even Initial Coin Offerings (ICOs). Due to the different types of funding levels, depending upon the month and types of funding rounds made, different orders of magnitude for investments between the months are seen. Therefore, in depicting the total amount of funding (in USD) for the period in Figure 5 , a logarithmic scale was used. The hype period can clearly be seen in the total amount of funding raised and the number of investments made, which are around an order of magnitude greater during the hype than before and after. It is worth noting that investments made include ICOs which allowed for the general public to 'easily' invest in various projects. Given the hype it is likely that quite a number of investments during the period were public investments fueled by nothing more than the hype. Given that Crunchbase feeds most of its investment-related data directly from its investor network, these results do not suffer from the lag seen in companies being listed in their dataset.

From the data it can be seen that whilst investments did peak during the hype period, they returned back to sustained pre-hype levels. This may indicate that indeed the sector has entered the Gartner hype cycle's Trough of Disillusionment and potentially on its way out towards the Slope of Enlightenment.

A similar exercise was conducted for investments reported on CB Insights depicted in Figure 6. Trend lines have been added to easily spot trends. Investments are seen to steadily increase till around the hype period, and thereafter tapers off, whilst indication of a potential increasement in investments is seen towards the end of 2020. This corroborates the findings above and may also be an indication of potential upcoming increased investment interest in the sector.

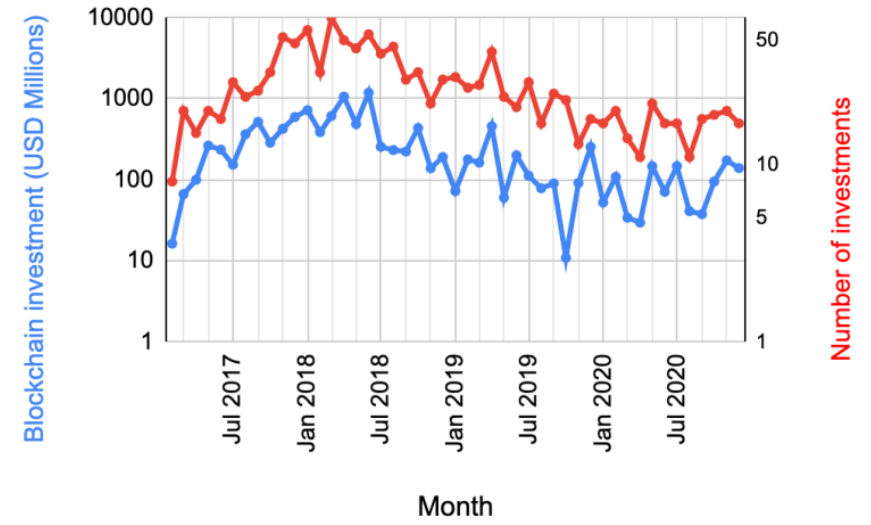

Figure 5: Investment raised (in USD) and number of investments made to Blockchain-related companies from Crunchbase.

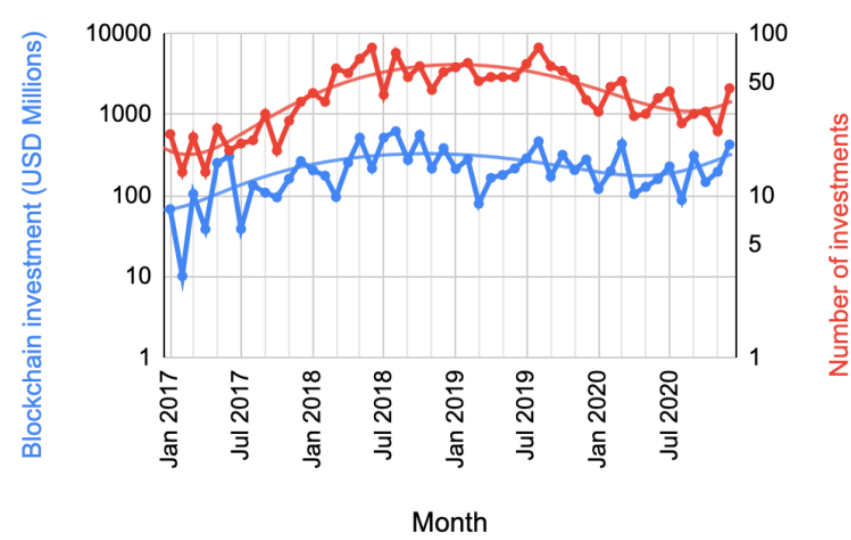

Figure 6: Investment raised (in USD) and number of investments made to Blockechain-related companies from CB Insights.

\section{Patents}

Patents, which provide companies with a manner to secure their intellectual property, can provide an indication in regard to innovation and development taking place, as well as to the sector's backing and investment to secure such innovation which can be quite costly and therefore demonstrates the sector's belief that investing in securing such intellectual rights will bear fruit in the future.

The number of patents registered (worldwide) per month between January 2017 and December 2020 was extracted from Espacenet $^{\mathrm{vi}}$ run by the European Patent Office (EPO). Espacenet was chosen as it reportedly has the highest number of patents in its database and has the "best features for searching" [9] when compared with Patentscope and Depatisnet - two other popular patent search engines.

\section{Methodology}

The number of patents published per month in the period were extracted using the following query format (this particular query extracts patents published in January 2017): (nftxt $=$ 'Blockechain' OR nftxt = 'Cryptocurrency' OR nftxt = 'Cryptocurrencies' OR nftxt $=$ 'Distributed Ledger Technology' 
OR nftxt $=$ 'Distributed Ledger Technologies' OR nftxt $=$ 'Bitcoin' OR nft xt $=$ 'Ethereum' OR nftxt $=$ 'Hyperledger' OR nftxt $=$ 'Smart contract' OR nftxt $=$ 'Smart contracts') AND pd within '2017-01-01, 2017-01-31'

The data was gathered on 14 January 2021.

\section{Results}

Patents can take quite some time until they are granted and published - even up to 'three to five years from the date'vii of application. Therefore, a lag will be seen in regard to a patent being granted and its publication. In fact, looking at Figure 7, which depicts the number of patents published on the subject matter per month, it can be seen that numbers substantially increase in November 2018 and continue to do so after. Further analysis has not been undertaken to determine whether this is due to a lag in patent publications post the 2017 hype period.

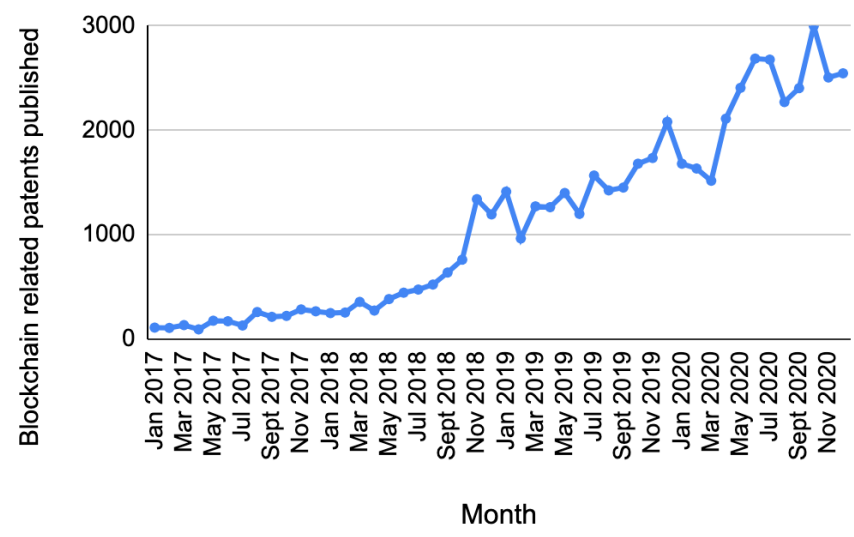

Figure 7: Blockechain-related patents published per month.

However, it can be noted that even if most of the patent publications granted in the more recent years were due to 2017's hype, the patent owners still saw utility in paying for the patent at the time of the grant/publication date (which is when majority of the patent registration costs are required to be paid).

Further analysis can be undertaken to determine whether a majority of the original dates of filing relate to the 2017 hype or not (though it does not seem likely from an initial glance).

Irrespective of this, we can draw the conclusion that increasingly more money is being spent on finalising patent publication as the months go by - which is an indication that the private sector still sees the domain to be one worth investing in.

\section{Academic Papers}

The current and future success of a technological sector is dependent not only on the private sector but also upon further research and development from the academic sector especially within an emerging sector such as this one.
To provide a picture of the academic interest within the sector, a primary output from academia, papers, have been investigated to provide an objective insight in regard to interest in the domain. The number of papers published each year has been extracted from the following popular academic paper indices and repositories which are known to have substantial overlap [10]: Google Scholar, ${ }^{\text {viii }}$ Web of Science, ${ }^{\text {ix }}$ Scopus $^{\mathrm{x}}$ and EBSCOxi (including all its databases). Whilst results heeded from Google Scholar may well include non-academic sources (including patents, technical reports, and other documents that the Google Scholar engine determines to be a paper), the Web of Science, Scopus and EBSCO repositories only index material which they deem to be trustworthy in terms of their academic relevance, and therefore it was decided to include the different repositories.

\section{Methodology}

The number of papers published were extracted over a period of a year. This was due to most of the databases providing a search criterion that enables for searching by granularity of a year and not of a finer granularity. The number of papers were extracted by searching for the same following terms and keywords within the papers across the different databases: Blockchain, Cryptocurrency, Cryptocurrencies, DLT, DLTs, 'Distributed Ledger Technology', 'Distributed Ledger Technologies', Bitcoin, Ethereum, Hyperledger, 'Smart contract', 'Smart contracts', 'Cryptocurrency exchange', 'Crypto exchange'. The results were extracted on 5 January 2021.

\section{Results}

Whilst the Web of Science, Scopus and EBSCO databases reported an exact number of papers that matched the search criteria, Google Scholar reported an indication of 'about' a number of resultant papers and therefore, the Google Scholar results are estimated values.

In Figure 8 a steady increase in the number of papers published can be seen across all the databases until 2019. Thereafter, for 2020, the number of papers reportedly published decrease slightly for Google Scholar, Web of Science and EBSCO, yet they are seen to continue to increase in the Scopus database.

Albeit at a slightly lower rate than the year before. This reported decrease in papers is likely not due to an actual dip in papers being published but due to the fact that the databases can take quite some time to be updated with published papers.

Google Scholar regularly adds 'new papers several times a week' yet it could take up to ' $6-9$ months to a year or longer' to update their records - and similarly the other databases can take a number of months to a year as well. 


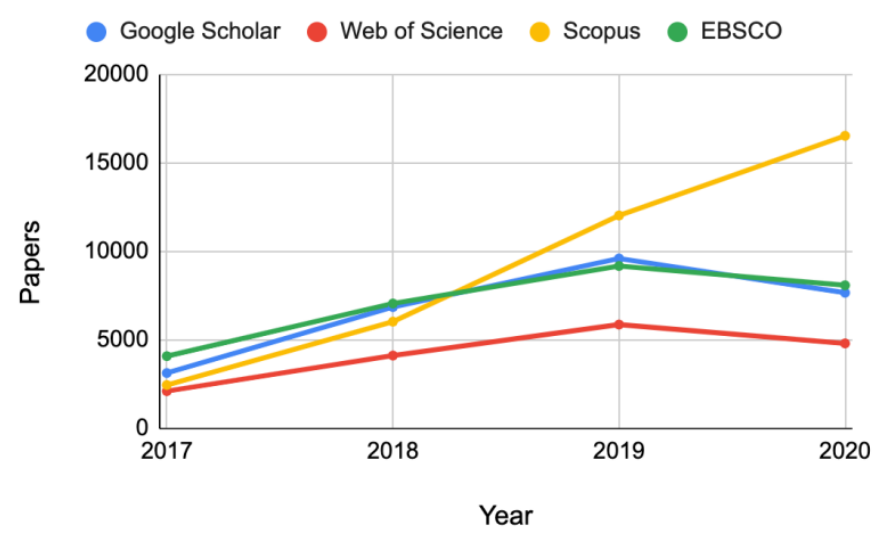

Figure 8: Blockechain-related papers published per year.

Therefore, these results should be revisited in a number of months to a year. Whilst, it cannot be definitely said that the number of papers published in 2020 increased compared to previous years, it is reasonable to assume that the number of papers will be roughly equal to 2019 if not more. Once enough time has passed to be able to get a full picture of 2020-related publications this can be confirmed. That being said the numbers indicate that there is not a significant dip in interest from the academic community, but in the least roughly the same level of interest. Whilst the rationale behind this exercise was to establish academic interest in blockchain (by surveying the numbers of papers published in the field), future work could be undertaken to establish how impactful the field's papers were (by looking at the number of times the papers have been cited).

\section{Research and Development Funding}

Academic papers can demonstrate academia's interest in the domain up till the recent past. Research and development funding can provide a picture of where academia and other research and development-based stakeholders will focus their time over the coming years. When funded by government it also provides an indication in regard to a government's support of a sector.

Research and development funding data was retrieved from the UK's national innovation agency, Innovate UK, xii in aim of determining governmental interest in the sector by comparing amounts of blockchain-related project funding over the years. Indeed, this data is only representative of a single funding agency from a single country (the UK), and further research should be undertaken to be able to draw global analysis.

\section{Methodology}

Innovate UK's public transparency dataset on their funded projects ${ }^{\mathrm{xiii}}$ was used and projects were filtered out so that only the ones whose description or title contained the following terms were included: Blockchain, Cryptocurrency, Cryptocurrencies, DLT, DLTs, 'Distributed Ledger Technology', 'Distributed Ledger Technologies', Bitcoin,
Ethereum, Hyperledger, 'Smart contract', 'Smart contracts', 'Cryptocurrency exchange', 'Crypto exchange'. The dataset also contains projects that were withdrawn, which were excluded from this analysis. The version uploaded on 8 January 2021 was usedxiv.

\section{Results}

Figure 9 provides a full view of the number of projects funded including the amount funded and starting date. The figure shows that 2019 and 2020 saw a substantial increase in the numbers of projects and size of projects funded. A number of these projects will be ongoing till August and November 2022. It can be assumed that many of these projects will contribute positively to the number of academic papers published within the domain. Indeed, again this is a single funding agency; however, the results are promising.

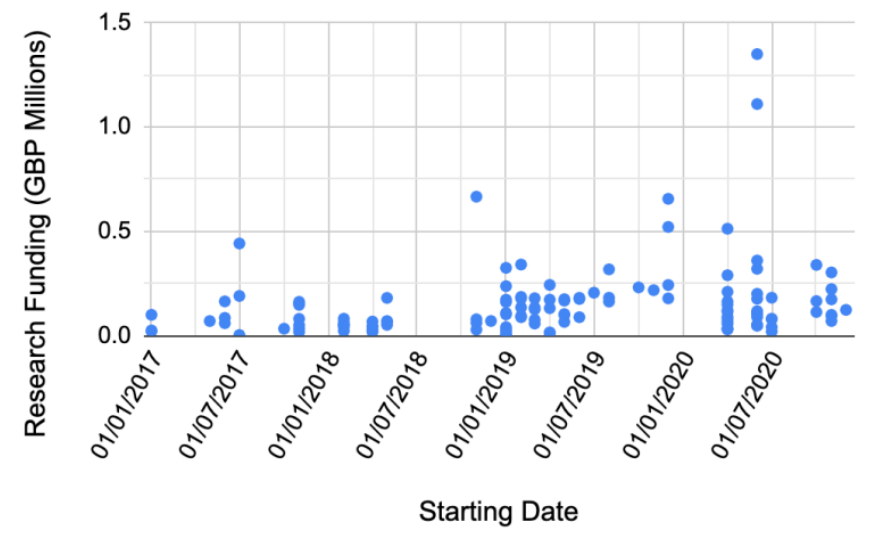

Figure 9: Innovate UK-funded blockchain-related research and development projects.

Figure 10 provides an overview for the different funding periods (2016/17 to 2019/20) that show total costs and grants issued for blockchain-related projects around doubling each year. The year 2019/20 saw a dip in awardees indicating largersized grants (as can be seen in Figure 9).

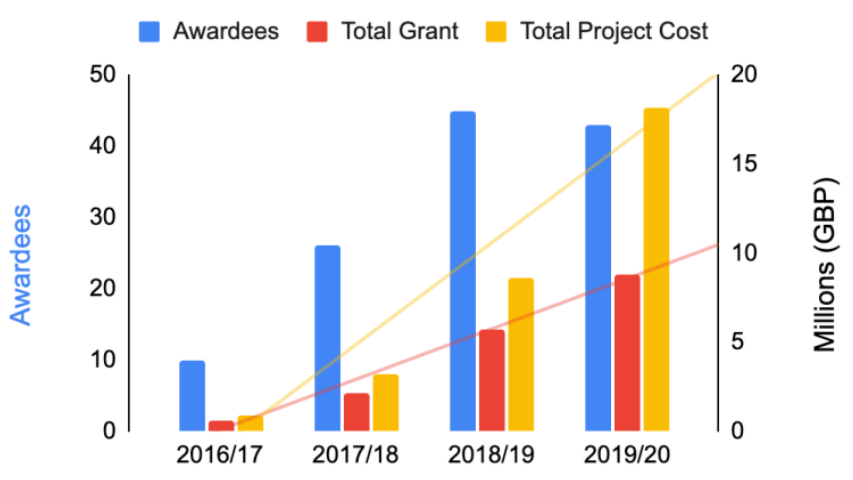

Academic Year

Figure 10: Innovate UK total blockchain-related project funding, costs and awardees. 


\section{Online Search Trends}

A technology's success is dependent not only on innovation and public and private sectors' support for that technology, but it must also be adopted by its main stakeholders - which may or may not be the general public. In the case of blockchain, DLT and its various applications, the level to which the general public may be interested in it widely varies. Nonetheless it would be useful to provide insight in regard to the varying interest and trends surrounding the various terms over the period. Even if a term is likely to be used by a small stakeholder group then its popularity over time should be representative of that stakeholder group (unless the term is used to mean something else or interest during some period is garnered by other groups).

Google Trends ${ }^{\mathrm{xv}}$ provides insight in respect to a search term or topic's search interest over time. A search term is the exact text that users type into Google's search engine, whilst a topic encapsulates many different search terms that Google deems to be categorised under the specific topic computed using 'an automated classification engine' [11]. The various terms/topic trend results are scaled to a percentage compared to all the other terms/topics that are used within the same trend results, as per the site's description: 'the resulting numbers are then scaled on a range of 0 to 100 based on a topic's proportion to all searches on all topics'xvi.

\section{Methodology}

Given that the results provided by Google Trends are scaled to a range of $0-100$, less popular terms/topic results end up being scaled down to $<1$ and even to 0 when compared with more popular terms/topics. Therefore, terms and topics were grouped together such that they would not result in scaling out relevant results. Whilst any search term can be used to generate trend results, topics are restricted to the ones that Google Trends has identified.

We gathered data for the following topics: Cryptocurrency, Bitcoin, Ethereum, Hyperledger, Smart contract and Distributed ledger; and for the following search terms: Blockchain, Bitcoin, Cryptocurrency, Cryptocurrencies, Ethereum, DLT, Hyperledger and 'Smart contract'. The results were retrieved for a Worldwide coverage of search popularity. The results were gathered on 5 January 2021.

\section{Results}

Bitcoin was the most popular topic and search term by a substantial difference. Figure 11 shows the Bitcoin topic and search time, along with the Cryptocurrency topic and Blockchain search term to be able to gauge the difference between them and the other topics and search terms discussed further below. The hype period in 2017 can clearly be seen, and both the Bitcoin topic and search term have practically the same results, except for a peak in the Bitcoin topic's trend during the beginning of September 2019. It is unclear why this topic has seen this peak and yet the search term itself does not see this increase in interest.

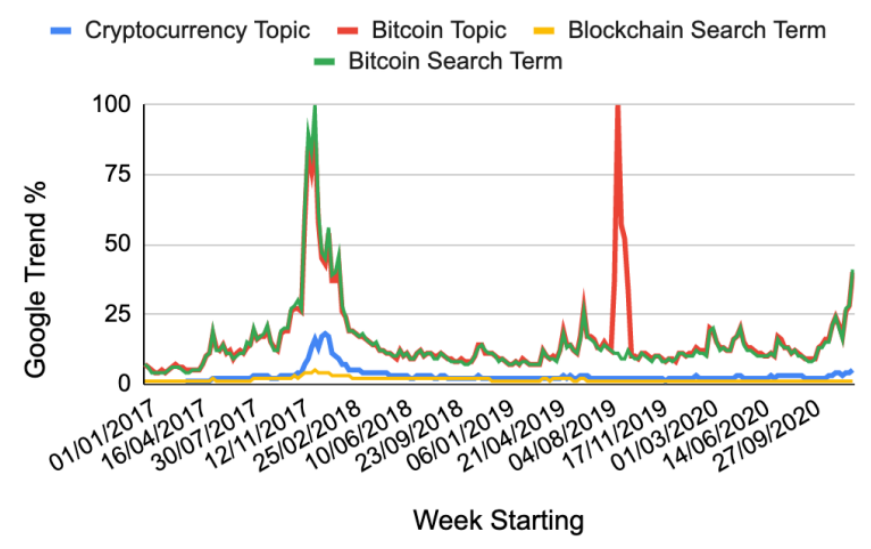

Figure 11: Google trend results for 'Cryptocurrency' and 'Bitcoin' topics, and 'Blockchain' and 'Bitcoin' search terms.

After the hype period, interest in Bitcoin is rather stable until the end of 2020 where the interest in Bitcoin can be seen to be peaking again - when Bitcoin's price started to peak again late 2020 (and eventually reach new all-time highs).

It is interesting to note that the Bitcoin search term and topic have substantially higher results than the other search terms and topics, indicating that the general public has been more interested in Bitcoin than the technology itself.

Results comparing the search terms Cryptocurrency, Blockchain and Ethereum are shown in Figure 12, whilst the results for the same topics are shown in Figure 13. Ethereum can be seen to peak during the beginning of the Initial Coin Offering (ICO) hype that started in Summer 2017, and then again in late 2017 when Bitcoin and cryptocurrencies in general had seen a peak of interest (and price surge).

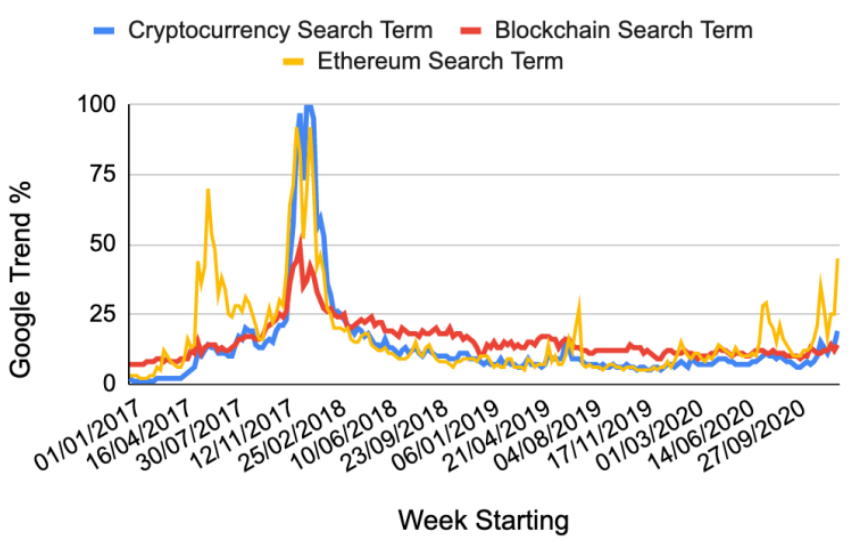

Figure 12: Google search term trend results for 'Cryptocurrency', 'Blockechain' and 'Ethereum'. 


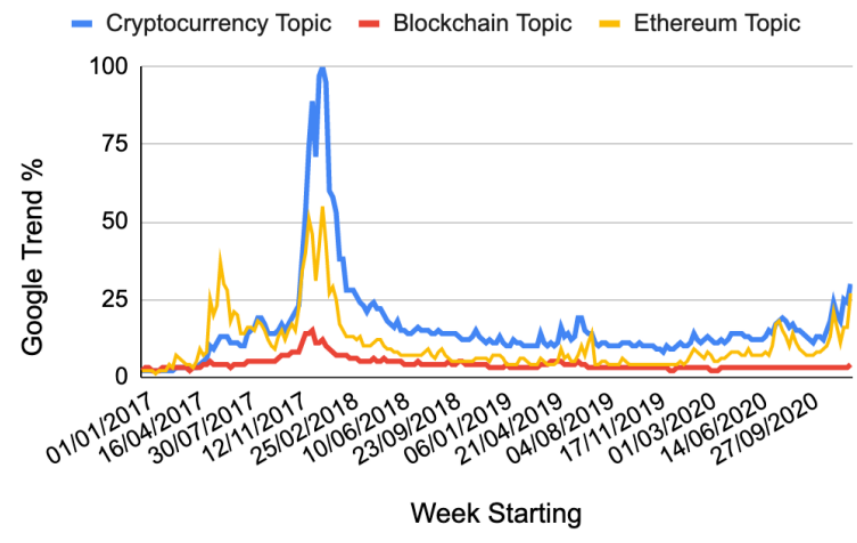

Figure 13: Google topic trend results for 'Cryptocurrency', 'Blockechain' and 'Ethereum'.

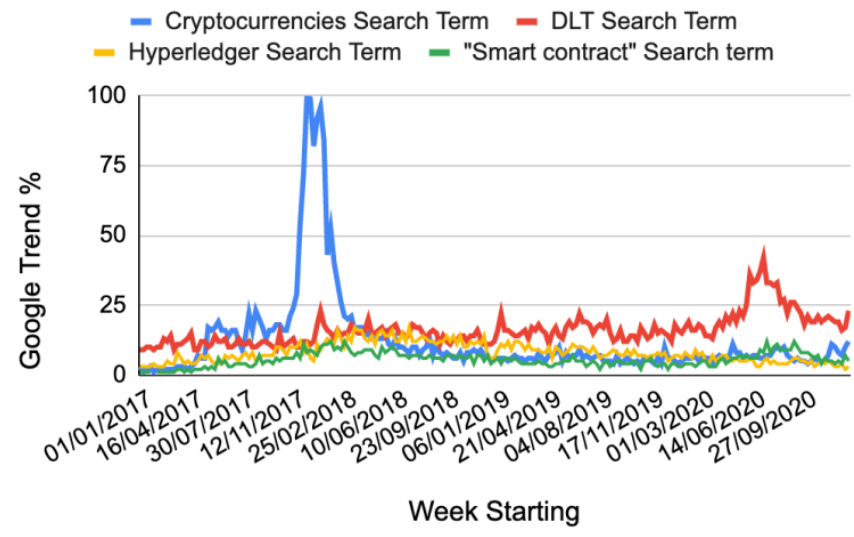

Figure 14: Google search term trend results for 'Cryptocurrencies', 'DLT', 'Hyperledger' and 'Smart contract'.

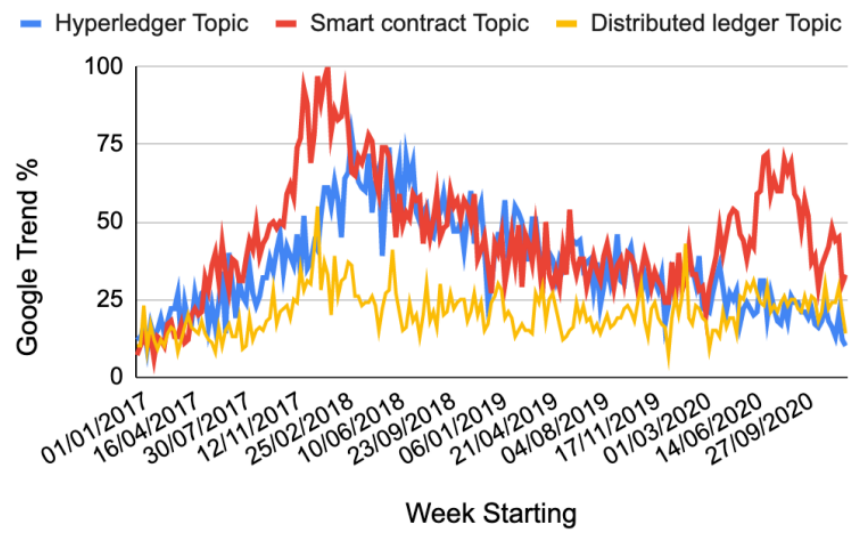

Figure 15: Google topic trend results for 'Hyperledger', 'Smart contract' and 'Distributed ledger'.

Figure 14 shows the trend results for the following search terms: Cryptocurrencies, DLT, Hyperledger and 'Smart contract'. Congruent with other results the cryptocurrencies term sees a peak of interest during the hype period in 2017. Smart contracts seem to increase in interest whilst it slightly lags after cryptocurrencies which could be due to specialists (developers and lawyers) wanting to learn more about the technology associated with the hype around cryptocurrencies.
Similarly, the DLT search term sees more interest as time passes which could be due to specialists' interest in the broader area of DLT.

In Figure 15 we can see more clearly the smart contract topic peak after the initial hype had started in mid-2017. Again, this may be due to specialists showing interest in the technology supporting the previous hype. Hyperledger, a popular blockchain infrastructure framework used typically for private blockchains can also be seen to garner interest after the mid2017 hype. The interest seems to peak in late 2018 and gradually diminish over time along with interest with smart contracts, though smart contracts see another peak of interest towards mid and end of 2020 .

From the various results we can conclude that interest in the various aspects remain at stable levels after the 2017 hype. Some of which see renewed interest potentially due to the increase in cryptocurrency price.

\section{Hash rate and Miners}

When it comes to operating a blockchain network, especially a proof-of-work-based one, the number of miners and computational power backing the network is a testament to the interest in the particular network as well as support for the network's success - as the more computational power, the less likely it becomes to successfully undertake an attack on the network. Here, an analysis of two of the most popular proofof-work-based cryptocurrency networks, Bitcoin and Ethereum, is provided.

Indeed, other consensus mechanisms are being proposed and used (such as proof-of-stake) which would be of interest to investigate; however, we leave this for future work.

\section{Methodology}

Hash rate datasets for the Bitcoin and Ethereum networks were retrieved from Coin Metricsxvii on 5 January 2021, for the period 1 January 2017 to 31 December 2020.

\section{Results}

Figure 16 depicts Bitcoin's and Ethereum's exahash per second and terrahash per second rates, respectively. Besides a slight dip in mid- to late 2018, Bitcoin shows steady growth in terms of computational power being put into the network. This dip likely occurred due to a number of miners deciding to stop mining as the price of bitcoin had reached its lowest point around that time, deeming the operation to not be profitable enough for some miners. Despite price fluctuations the amount of computational power in the Bitcoin network sees steady growth which is an indicator that the number of miners and/or the amount of resources they are putting behind the network is increasing which is a testament to miners' and the network's success in spite of any claimed inefficiencies [12]. Ethereum sees a similar trend though on a smaller terrahash scale. 


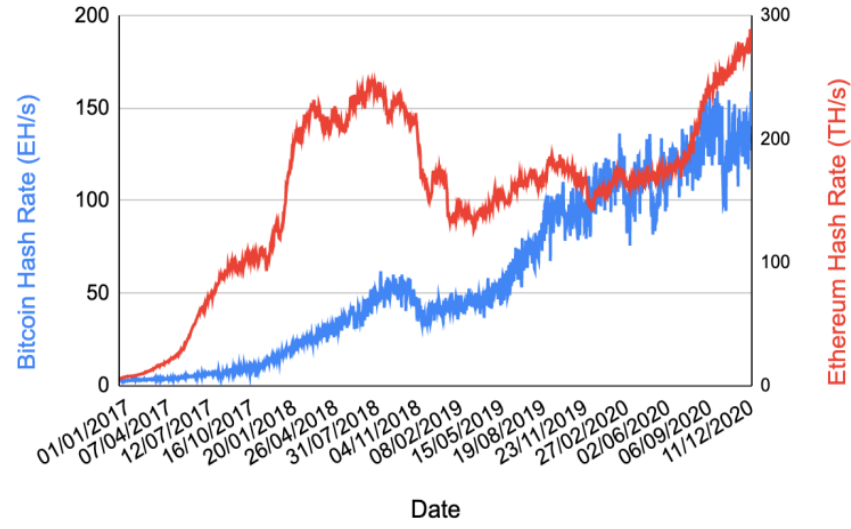

Figure 16: Bitcoin and Ethereum hash rates.

\section{Code repository activity and Software developers}

Having looked into various stakeholders from the private and public sectors, to academia and the general public to the miners supporting such networks, it would be ideal to provide insight in regard to an indication of activity amongst the software developers creating the technology. GitHubxvii is a popular code repository used by software developers around the world for both open-source software projects as well as private repositories.

To establish whether blockchain software development activity is deemed to be dead or alive, activity of open-source GitHub code repositories were analysed.

\section{Methodology}

GH Archivexix provides an up-to-date archive of all activitive that take place in public GitHub code repositories. The $\mathrm{GH}$ Archive data required was retrieved using the Google BigQuery dataset provided. To make use of the data, though, it was required to determine which projects were relevant.

The GitHub REST APIxx was used to retrieve repositories that were categorised under the 'blockchain' topic. A total of 11,893 repositories were categorised under the blockchain topic (as retrieved using the API). The API, however, only allows for retrieval of 1,000 results per search query. Therefore, a script was written to return results in batches of a maximum of 1,000 results for distinct queries. Searches were repeated for repositories containing words starting with ' $a$ ' to ' $z$ ' and ' 0 ' to ' 9 '. To gather a larger list of projects the process was repeated for the different combinations of the second letter of a word contained within a project's name. Ultimately, this resulted in retrieval of the names of 11,605 distinct projects categorised under the blockchain topic. This means that a remaining 288 blockchain project names were not retrieved - yet just over $97 \%$ of project names were retrieved. In the interest of time, it was decided that enough data had been collected to undertake an initial investigation.
Using the list of blockchain-related project names retrieved using the GitHub REST API, the GH Archive was then used to extract the number of events that took place on the relevant projects per month between January 2017 and December 2020. All repository events and types were included. The types of events ${ }^{\mathrm{xxi}}$ are: PushEvent, IssueCommentEvent, IssuesEvent, CreateEvent, WatchEvent, MemberEvent, CommitCommentEvent, PullRequestEvent, DeleteEvent, ForkEvent, PullRequestReviewCommentEvent, PublicEvent, ReleaseEvent and GollumEvent.

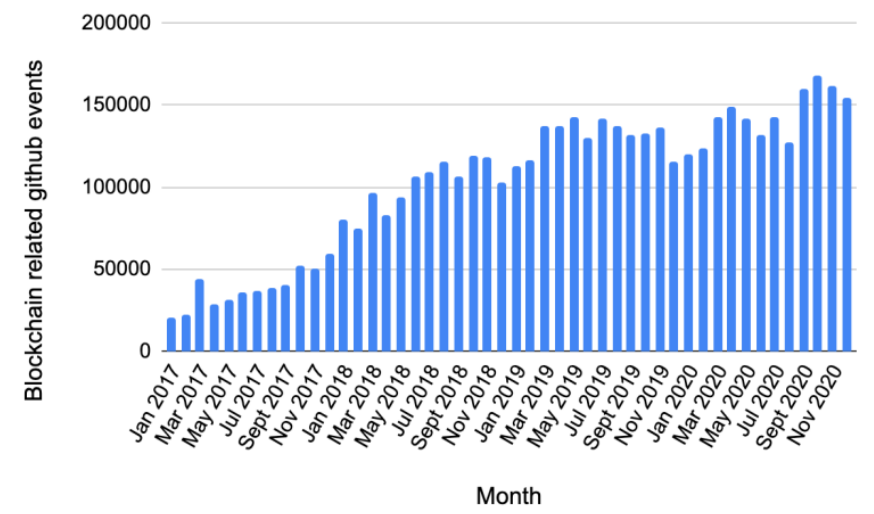

Figure 17: GitHub Blockchain-related code repository events.

\section{Results}

Figure 17 shows the total number of blockchain code repository events per month between January 2017 and December 2020. The figure shows a steady increase in activity across the different blockchain-related GitHub code repositories which indicates that the amount of work in developing and maintaining blockchain-related projects is ever increasing with time. Interestingly, effects pertaining to hype and/or related cryptocurrency pricing cannot be seen to affect the total development effort across the different projects. Blockchain-related development is far from dead, and seems to not be affected by neither surges nor drops in related cryptocurrency prices.

Whilst, it is very hard to determine why individual projects may survive or not since there are many different external factors at play, it would be ideal to identify traits of successful projects. Figure 18 depicts the number of contributors to a project against the number of days since a project was last active, and how long a project remained active for. As suggested in [13] the duration of activity is calculated as the difference between its first and last repository activity event.

Whether a project that is likely to be successful attracts more contributors, or whether having more contributors is more likely to make a project successful is hard to tell - yet it can be seen that the more contributors a project has the more active it is (i.e. around 0 days since the last activity) and the longer the project was/is still alive for. 


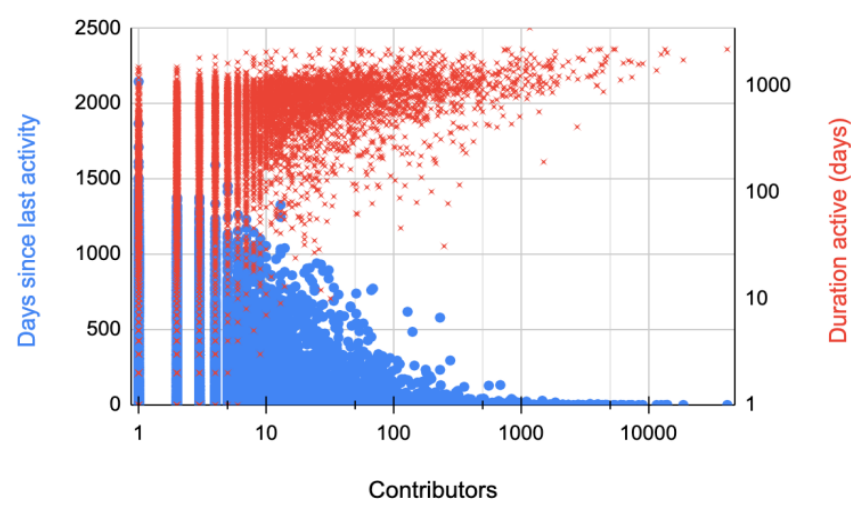

Figure 18: Number of GitHub Blockechain-related project contributors compared to the number of days since the projects' last activity and the project active lifetime.

\section{Conclusions}

Is Blockchain dead? We started off with the question, and in aim of answering it we investigated various aspects of the sector which may or may not provide insight in regard to whether it is dead or alive. Points to highlight from above include:

(i) Whilst the number of companies founded per month has decreased after the hype period according to a popular tech start-up/company registry (Crunchbase), the decreasing number of companies founded for a broader 'software' sector also decrease per month. An explanation behind this is congruent with claims that companies may not necessarily be registered on the site in their early stages until they desire to be listed or have already managed to attract investment. These results should be revisited in future to see if the numbers of companies founded in Crunchbase for 2019 and 2020 increase to support this claim;

(ii) The 2017 hype saw an increase in investment in blockchain-related start-ups and companies. Though posthype investment levels did drop slightly, they still remained at a stable level;

(iii) Patents published has steadily increased since 2017. Whilst patents do take time to be granted and published, the main granting and publication costs are paid at the end which means in the very least patent owners are still willing to invest substantial cost to secure their patents (which may be from a few years before), or that more patents are being submitted as time goes by;

(iv) The number of academic papers has been steadily increasing since 2017 and reported numbers drop slightly for 2020. However, as discussed, academic databases and indices can take up to a number of months and even up to year to include some papers. Therefore, given that this paper was written at the very beginning of 2021, it is likely that a large number of papers had not been included yet in the reported numbers. Therefore, these figures should be revisited in future to see if the number of papers reportedly published for 2020 increase to more than that of 2019. Nonetheless, even if the slight drop in papers turns out to be the reality, the numbers are still stable;

(v) Whilst data from only one governmental research and development funding agency was investigated and future investigation on other agencies around the world should be looked into, the amount of investment in blockchainrelated projects can be seen to increase year on end;

(vi) Public interest determined by search engine results pertaining to the sector may very well be swayed by hype as seen in the data presented herein. However, stable interest in the sector remains post-hype. The public, according to the search trend results, is generally more interested in cryptocurrency than blockchain technology. It may be deemed that there is a need for a stronger educational drive with respect to the technology and the benefits it provides beyond cryptocurrencies;

(vii) Interest in mining for popular cryptocurrency blockchain networks, Bitcoin and Ethereum, can be seen to increase steadily - a testament to the success of both miners and the network as a whole;

(viii)Software development effort is steadily increasing, without detriment from fluctuating currencies or hype, over time. A trait that can be seen from the data is that the more contributors a project has, the longer the project has lasted and also, the least amount of time has passed since the last activity was undertaken. This seems to be a case of strength in numbers. Projects of isolated developers may be less likely to succeed. Future work should be undertaken to establish whether collaboration, partnerships and/or code reuse between different projects provides any indication towards a project's success in staying alive.

Indeed, this work only provides an initial investigation into the various facets determining their activity. However, going into more depth in the various aspects is left as future work. Various results from this study and future work will be disseminated online at http://blockchainthings.io

From the analysis undertaken it can be concluded that either: if blockchain is dead then substantial private investment continues to be made in vain, money is being wasted on securing intellectual property that is not worth the costs, academics are busy undertaking research and publishing papers in a field that is doomed, government money is being spent on furthering research and innovation that will not result in advancement, substantial stakeholders and the general public are still busy searching online in the domain finding information that will likely be irrelevant soon, funds are being spent in mining infrastructure to secure and support a network that will cease to exist as well as to make profits in a 
cryptocurrency that will be worthless, endless hours are being spent in developing software that will not be used; or blockechain is not dead. Long live Blockchain!

Competing Interests:

None declared.

Ethical approval:

Not applicable.

Author's contribution:

Confirmed that I am the sole author responsible for all aspects of the paper.

Funding:

None declared.

\section{Acknowledgements:}

I would like to thank the team at the Centre for Distributed Ledger Technologies at the University of Malta, and all the Masters in Blockechain and DLT lecturers, and past and present students for engaging in various discussions that had lead me to undertake this study, particularly Prof. Gordon I. Pace with whom I have spent countless hours debating various related topics.

\section{References:}

[1] M. Crosby, Nachiappan, P. Pattanayak, S. Verma, and V. Kalyanaraman, "Blockchain Technology - BEYOND BITCOIN," Berkley Eng., 2016.

[2] J. Atherton, "Who is the Blockchain Employee? Exploring Skills in Demand using Observations from the Australian Labour Market and Behavioural Institutional Cryptoeconomics," J. Br. Blockechain Assoc., vol. 3, no. 2, pp. 1-12, 2020, doi: 10.31585/jbba-3-2-(4)2020.

[3] J.-M. Dalle, M. den Besten, and C. Menon, "Using Crunchbase for economic and managerial research," OECD Sci. Technol. Ind. Work. Pap., 2017, doi: https://doi.org/10.1787/18151965.

[4] F. Ferrati and M. Muffatto, "Using crunchbase for research in entrepreneurship: Data content and structure," Proc. Eur. Conf. Res. Methods Bus. Manag. Stud., vol. 2020-June, no. July 2007, pp. 342-351, 2020, doi: 10.34190/ERM.20.120.

[5] N. Naqvi, "Evidence-Based Blockchain: Findings from a Global Study of Blockchain Projects and Start-up Companies," J. Br. Blockchain Assoc., vol. 3, no. 2, 2020, doi: 10.31585/ibba-3-2-(8)2020.

[6] G. Fridgen, J. Lockl, S. Radszuwill, A. Rieger, A. Schweizer, and N. Urbach, "A solution in search of a problem: A method for the development of blockchain use cases," 2018.

[7] G. Tarasconi and C. Menon, "Matching Crunchbase with patent data," OECD Sci. Technol. Ind. Work. Pap., 2017.

[8] C. Lougen, "CB Insights," Journal of Business and Finance Librarianship, vol. 22, no. 3-4. 2017, doi: 10.1080/08963568.2017.1372018.

[9] B. Jürgens and V. Herrero-Solana, "Espacenet, Patentscope and Depatisnet: A comparison approach," World Pat. Inf., vol. 42, pp. 4-12, 2015, doi: 10.1016/j.wpi.2015.05.004.

[10] A. Martín-Martín, E. Orduna-Malea, M. Thelwall, and E. Delgado López-Cózar, "Google Scholar, Web of Science, and Scopus: A systematic comparison of citations in 252 subject categories," J. Informetr., vol. 12, no. 4, pp. 11601177, 2018, doi: 10.1016/j.joi.2018.09.002.
[11] S. Vosen and T. Schmidt, "Forecasting private consumption: Survey-based indicators vs. Google trends," J. Forecast., vol. 30, no. 6, pp. 565-578, 2011, doi: 10.1002/for.1213.

[12] A. Urquhart, "The inefficiency of Bitcoin," Econ. Lett., vol. 148, pp. 80-82, 2016, doi:

10.1016/j.econlet.2016.09.019.

[13] E. Kalliamvakou, L. Singer, G. Gousios, D. M. German, K. Blincoe, and D. Damian, "The promises and perils of mining GitHub," 11th Work. Conf. Min. Softw. Repos. MSR 2014 - Proc., pp. 92-101, 2014, doi: $10.1145 / 2597073.2597074$.

i https://www.bloomberg.com/news/articles/2019-11-

$12 /$ blockchain-is-dead-crypto-geeks-debate-merits-of-once-deartech

ii https://www.gartner.com/en/documents/3987450/hype-cyclefor-blockchain-technologies-2020

iii http://www.crunchbase.com

iv https://www.businessinsider.com/whither-techcrunch-2011-9 accessed on 12th January 2021.

v https://www.cbinsights.com/

vi https://worldwide.espacenet.com/

vii https://www.epo.org/service-support/faq/procedure-law.html viii https://scholar.google.com/

ix https://webofknowledge.com/

x https://www.scopus.com/

xi https://www.ebsco.com/

xii https://www.gov.uk/government/organisations/innovate-uk

xiii https://www.gov.uk/government/publications/innovate-uk-

funded-projects

xiv Ibid.

xv https://trends.google.com/

xvi https://support.google.com/trends/answer/4365533?hl=en

xvii https://coinmetrics.io/community-network-data/

xviii https://github.com/

xix https://www.gharchive.org/

xx https://docs.github.com/en/rest

xxi https://docs.github.com/en/developers/webhooks-andevents/github-event-types 